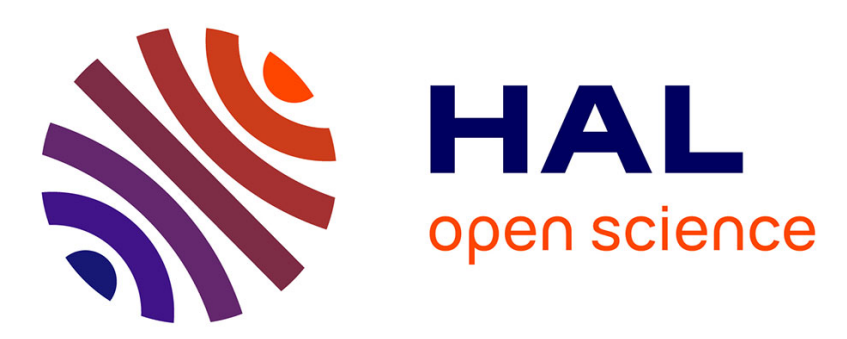

\title{
Analysis of the RATAN-600 radiotelescope antenna with a multilevel physical optics algorithm
}

\author{
Christine Letrou, Vladimir B. Khaikin, Amir Boag
}

\section{To cite this version:}

Christine Letrou, Vladimir B. Khaikin, Amir Boag. Analysis of the RATAN-600 radiotelescope antenna with a multilevel physical optics algorithm. Comptes Rendus. Physique, 2012, 13 (1), pp.38-45. 10.1016/j.crhy.2011.10.011 . hal-00726148

\section{HAL Id: hal-00726148 https://hal.science/hal-00726148}

Submitted on 29 Aug 2012

HAL is a multi-disciplinary open access archive for the deposit and dissemination of scientific research documents, whether they are published or not. The documents may come from teaching and research institutions in France or abroad, or from public or private research centers.
L'archive ouverte pluridisciplinaire HAL, est destinée au dépôt et à la diffusion de documents scientifiques de niveau recherche, publiés ou non, émanant des établissements d'enseignement et de recherche français ou étrangers, des laboratoires publics ou privés. 
The next generation radiotelescopes/ Les radiotélescopes du futur

\title{
Analysis of the RATAN-600 radiotelescope antenna with a multilevel Physical Optics algorithm
}

\author{
Christine Letrou ${ }^{\mathrm{a}}$, Vladimir Khaikin ${ }^{\mathrm{b}}$, Amir Boag ${ }^{\mathrm{c}}$ \\ a TELECOM SudParis/Lab. SAMOVAR (UMR CNRS 5157), F-91011 Evry Cedex, France \\ b The Special Astrophysical Observatory, RAS, Russia \\ ${ }^{\mathrm{c}}$ School of Electrical Engineering, Tel-Aviv University, Tel-Aviv, Israel
}

\begin{abstract}
The RATAN-600 antenna is a flexible multireflector system composed of reflectors of very large dimensions. An extended system, with improved performance in the millimetric range, includes a focal receiver array. Accurate electromagnetic analysis of such a system, and simulation of three-dimensional (3D) patterns, represents a substantial computational challenge. A fast Physical Optics method based on a multilevel subdivision of the surfaces of integration is proposed to address this problem. This method allows to perform Physical Optics integrals with a computational complexity comparable to that of the Fast Fourier Transform. The algorithm and initial numerical results of its application to the RATAN-600 antenna system are presented. To cite this article: C. Letrou, V. Khaikin, A. Boag, C. R. Physique 13 (2012).

\section{Résumé}

Analyse de l'antenne du radiotélescope RATAN-600 par une méthode d'Optique Physique multiniveaux. L'antenne du radiotélescope RATAN-600 est un système flexible composé de plusieurs réflecteurs, dont certains de très grandes dimensions. Dans sa configuration la plus performante pour le domaine millimétrique, elle est dotée d'un réseau focal. Un tel système constitue un défi du point de vue de l'analyse électromagnétique, si l'on souhaite disposer de résultats précis sur son diagramme dans l'espace en 3D. Une méthode d'Optique Physique rapide basée sur une décomposition multi-niveaux des surfaces d'intégration, permettant de rendre la complexité des calculs d'intégrales comparable à celle de transformées de Fourier rapides, est proposée pour répondre à ce besoin. L'algorithme de la méthode est présenté, ainsi que son application au cas du RATAN-600 et des résultats numériques. Pour citer cet article : C. Letrou, V. Khaikin, A. Boag, C. R. Physique 13 (2012).
\end{abstract}

Key words: Radiotelescope; Multireflector antenna; Physical Optics ; Multilevel algorithm

Mots-clés : Radiotélescope; Antenne multiréflecteurs; Optique Physique; Algorithme multi-niveaux

Email addresses: christine.letrou@it-sudparis.eu (Christine Letrou), vkhstu@mail.ru (Vladimir Khaikin), boag@eng.tau.ac.il (Amir Boag). 


\section{Introduction}

The RATAN-600 radiotelescope is based on a variable profile antenna in which the main mirror/reflector shape depends on the cosmic source elevation [1,2]. The beam pattern of RATAN-600 scans the space not by rotation of the whole reflector but by changing the shape of the main mirror and by moving the secondary mirror on a radial railway. The main mirror is basically an elliptic conus section, with an average diameter of 576 meters, parts of which can be transformed into parabolic cylinder and circular conus sections, depending on the elevation angle of observation (around $0^{\circ}$ and $90^{\circ}$ respectively). This telescope has been designed for observations in the $600 \mathrm{MHz}$ to $30 \mathrm{GHz}$ frequency range. This range is being extended into the millimetric frequency domain, more specifically to the $33-38 \mathrm{GHz}$ and $41-44 \mathrm{GHz}$ frequency bands (see http://www.spbf.sao.ru/CG/ for more details).

The optical scheme of the antenna system in the "South sector + Flat reflector" operating mode (S+F system) is shown in Fig. 1. The flat reflector is used as a periscope to redirect waves horizontally towards the primary mirror (also called "main reflector" in the following). This reflector is configured into a parabolic cylindrical surface perpendicular to the horizon, that provides focusing of the incoming waves in the horizontal plane (Fig. 2). The secondary mirror has the shape of a parabolic cylindrical surface, whose generatrix is parallel to the horizon, and it focuses the waves in the vertical plane at a distance of $2150 \mathrm{~mm}$ from its vertex (Fig. 3). The main reflector settings are such that the focal points in the horizontal and vertical planes coincide. An extended version of this $\mathrm{S}+\mathrm{F}$ system, based on the use of

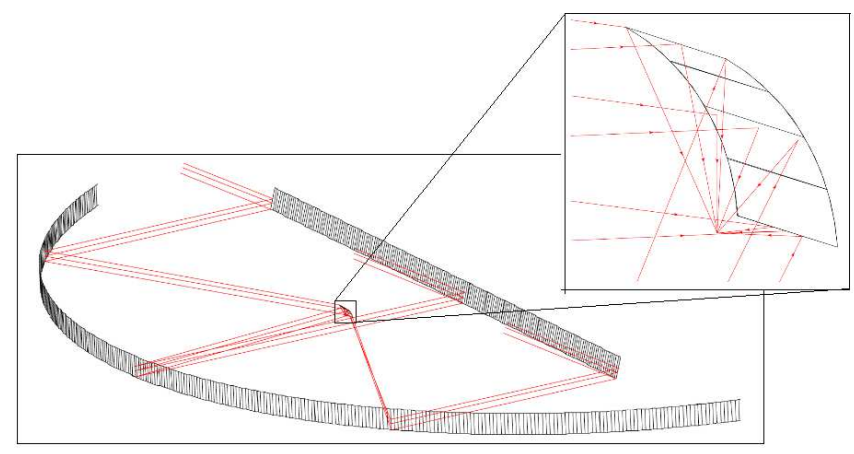

Figure 1. Schematic 3D view of the RATAN-600 radiotelescope $\mathrm{S}+\mathrm{F}$ antenna system. In red: some ray trajectories.

a focal receiver array, is currently under study to enlarge the observed area in the sky at millimetric wavelengths. Such an extension would be of particular interest for observations of Cosmic Background anisotropies. To minimize wave aberrations while extending the Field of View of the telescope, a longfocus optics has been suggested in the form of a small tertiary quasi-elliptic mirror with double curvature (not a figure of rotation) installed close to the secondary focus [3].

Analysis of the RATAN-600 S+F antenna system at millimeter-wave frequencies represents a substantial computational challenge, due to its very large electrical dimensions: the main reflector surface area is of about 40 millions of wavelengths for observations conducted at the $\lambda=8 \mathrm{~mm}$ wavelength. Until recently, this quasi-optical system has been mainly analyzed using Geometrical Optics (GO)/ray-tracing techniques combined with the aperture integration method [3]. However, GO analysis does not provide sufficient information on near-field diffraction effects and other wave related phenomena, in a context where reflectors are not in their mutual far field regions, as can be inferred from the dimensions given in Figs. 2 and 3. This point is even more critical in the case of the extended S+F system, where the tertiary reflector potentially generates new diffraction and cross-polarization effects. Also, the aperture integration method may only be used for calculating the main beam and the nearest sidelobes. An attempt was made 


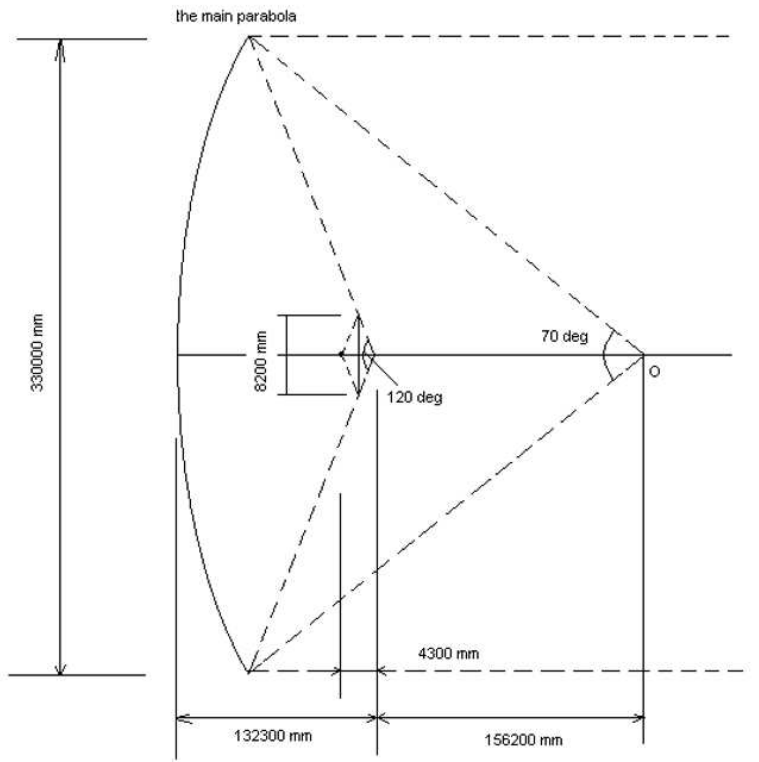

Figure 2. Horizontal cut of the primary and secondary reflectors of the RATAN-600 radiotelescope (South sector).

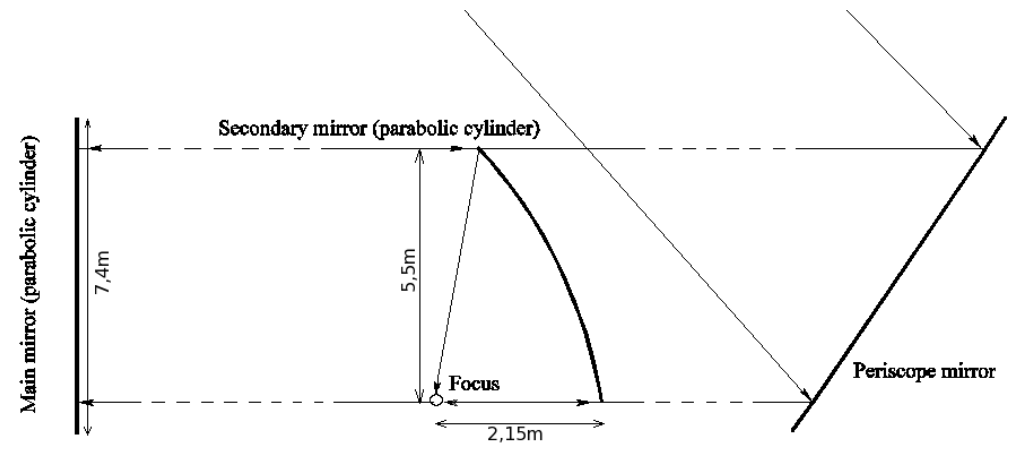

Figure 3. Vertical cut of the RATAN-600 radiotelescope $\mathrm{S}+\mathrm{F}$ antenna system. Distances between the reflectors are not scaled.

to take diffraction effects into account via Fresnel integrals, with a particular interest for wavelengths larger than 3 centimeters [4].

The Physical Optics (PO) approximation is of current use for reflector antenna systems analysis, appearing as a good compromise between the computational feasibility and accuracy requirements. The "non far field" version of PO surface integrals, complemented with the contribution of Physical Theory of Diffraction line integrals, can address multireflector problems with sufficient accuracy on the whole sphere of directions in the three-dimensional space [5]. In the straightforward implementations of the PO algorithm, the computational cost of surface integrals is of the order of $O\left(N^{4}\right)$ with $N$ being the linear dimension of the radiating surface with respect to the wavelength. For large reflector electrical dimensions such implementations lead to excessively long computation times. This is especially true when repeated evaluation of the radiation patterns is required for antenna geometry optimization with multiple primary sources. 
The fast Multilevel Physical Optics (MLPO) algorithm was introduced in [6] in order to reduce the complexity of evaluating the PO integrals over arbitrary shaped surfaces to a level comparable to that of the FFT-based techniques, i.e. $O\left(N^{2} \log N\right)$. To analyze the RATAN-600 S+F antenna system, the conventional MLPO algorithm has been generalized in order to cope with oblong rectangular reflector shapes, and with complex systems involving more than two reflectors. The "non far field " formulation of the PO integrals is used to compute fields radiated by one reflector surface to the other one.

In this paper, we first present the formulation of the conventional MLPO algorithm, used for far field patterns computations (Section 2). Section 3 is then devoted to the formulation of the generalized MLPO algorithm used for the RATAN-600 S+F antenna system analysis. Finally, Section 4 presents first numerical results obtained for the analysis of this system (without the long focus extension).

\section{Conventional MLPO algorithm}

This section first introduces the far field formulation of Physical Optics (Subsection 2.1), and recalls some properties of radiation patterns which are used intensively by the MLPO algorithm (Subsection 2.2). The conventional MLPO algorithm is described in subsection 2.3.

\subsection{Far field formulation}

Although PO and MLPO have also demonstrated their ability to evaluate the radiation patterns of dielectric structures, we shall consider here the case of a perfectly conducting surface $S$ illuminated by incident fields, which is of direct interest in view of future analysis of the RATAN-600 antenna system. The incident fields create on $S$ a magnetic field distribution denoted by $\boldsymbol{H}^{\mathrm{s}}\left(\boldsymbol{r}^{\mathrm{s}}\right), \boldsymbol{r}^{\mathrm{s}} \in S$.

The radiation pattern $\boldsymbol{U}(\theta, \phi)$ is defined via the far field $\boldsymbol{E}(\boldsymbol{r})$ as

$$
\boldsymbol{U}(\theta, \phi)=4 \pi r e^{j k r} \boldsymbol{E}(\boldsymbol{r})
$$

where $(r, \theta, \phi)$ are the spherical coordinates of the observation point $\boldsymbol{r}$ and $k=2 \pi f / c$ is the wavenumber, with $c$ being the speed of light and $f$ the frequency. Harmonic time dependence $e^{j \omega t}, \omega=2 \pi f$, is assumed and the corresponding term is suppressed in the equations.

In the Physical Optics approximation, the radiation pattern is computed as an integral transform of the field over the reflector surface:

$$
\boldsymbol{U}(\theta, \phi)=j k \widehat{\boldsymbol{r}} \times \int_{S} 2 \eta \widehat{\boldsymbol{r}} \times\left(\widehat{\boldsymbol{n}}^{\mathrm{s}} \times \boldsymbol{H}^{\mathrm{s}}\left(\boldsymbol{r}^{\mathrm{s}}\right)\right) e^{j k \widehat{\boldsymbol{r}} \cdot \boldsymbol{r}^{\mathrm{s}}} d s
$$

with $\widehat{\boldsymbol{n}}^{\mathrm{s}}$ being a unit vector outward normal to $S$ at point $\boldsymbol{r}^{\mathrm{s}}, \widehat{\boldsymbol{r}}(\theta, \phi)=(\sin \theta \cos \phi, \sin \theta \sin \phi, \cos \theta)$ the unit vector in the direction of observation, and $\eta$ the free space wave impedance. For the sake of simplicity, we shall not introduce in this paper the Physical Theory of Diffraction (PTD) contribution, which is obtained by line integrals along the rim of the radiating surface, and easily fits into the MLPO algorithm. The reader is referred to [7] which gives details about this contribution and numerical results illustrating the accuracy and efficiency of 3D pattern computations obtained with the MLPO including the PTD integrals.

\subsection{Radiation pattern properties}

Property 1: Radiation pattern sampling [8,9]

The electrical size of a radiating surface $S$ is defined as $N=k R$ with $R$ being the radius of its smallest circumscribing sphere. The far field pattern radiated by $S$ is expressed as a function of the spherical 
angular coordinates, $\theta$ and $\phi$, defining the radiation directions $\hat{\boldsymbol{r}}=(\sin \theta \cos \phi, \sin \theta \sin \phi, \cos \theta)$ in a given coordinate system $\mathcal{S}$. If $\mathcal{S}$ has its origin at the center of the smallest sphere circumscribing $S$, this pattern is sufficiently sampled along the angular variable $\beta(\beta=\theta$ or $\phi)$ by sampling the observation range along $\beta, \beta_{\max }-\beta_{\min }$, with the number of points:

$$
N_{\beta}(N)=\left\lceil\Omega N\left(\beta_{\max }-\beta_{\min }\right) / \pi\right\rceil+C_{\beta}
$$

where \lceil\rceil denotes "the smallest integer larger than", $\Omega>1$ is an oversampling factor, and $C_{\beta}$ the minimum number of points which guarantees a sufficient sampling of infinitesimal source patterns.

\section{Property 2: Expression of a far field pattern in a translated coordinate system}

Let us denote $\mathcal{S}$ a coordinate system with origin $O$ and $\mathcal{S}^{\prime}$ a coordinate system with origin $O^{\prime}$ obtained by translating $\mathcal{S}$. Let $\boldsymbol{U}(\hat{\boldsymbol{r}})$ and $\boldsymbol{U}^{\prime}(\hat{\boldsymbol{r}})$, denote the far field patterns of a radiating object in coordinate systems $\mathcal{S}$ and $\mathcal{S}^{\prime}$, respectively, with a given $\hat{\boldsymbol{r}}$ defining the same observation direction in both coordinate systems (far field approximation). Then: $\boldsymbol{U}^{\prime}(\hat{\boldsymbol{r}})=\boldsymbol{U}(\hat{\boldsymbol{r}}) \exp \left(-j k \hat{\boldsymbol{r}} \cdot \overrightarrow{O O^{\prime}}\right)$.

\subsection{MLPO algorithm}

The MLPO algorithm takes advantage of Property 1 by replacing a PO surface integration on a large surface by PO integrals on small subdomain surfaces and subsequent aggregation of the elemental patterns of these small subdomains. To this end, a multilevel hierarchical subdivision of the radiating surface of interest into subdomains of decreasing size is performed, until the subdomain size is of the order of the wavelength.

The hierarchical subdivision of the radiating surface $S$ is performed from level $L=0$ (no subdivision) to level $L=M$ (smallest subdomains). A surface subdomain obtained after $L$ subdivisions of the surface is denoted by $S_{n}^{L}, n=1$ to $N_{\mathrm{s}}^{L}$, with $N_{\mathrm{s}}^{L}$ the number of surface subdomains at level $L$. The subdivision process at level $L$ subdivides each subdomain $S_{m}^{L-1}$ of level $L-1$ into smaller subdomains $S_{n}^{L}$. In the following, $S_{m}^{L-1}$ will be called the "parent" of the $S_{n}^{L}$ resulting from its subdivision. In a binary scheme, the electrical size $N=k R_{n}^{L}$ of a level $L$ subdomain $S_{n}^{L}$ is approximately equal to one half of the size $k R_{m}^{L-1}$ of its parent of level $L-1$.

For reflectors whose surface projects onto a rectangle (resp. a disk) in a plane perpendicular to the antenna axis, the natural choice is to parameterize the surface with cartesian (resp. polar) coordinates in that plane. In the conventional MLPO algorithm, the hierarchical surface subdivision is performed according to a binary scheme. When the reflector surface projects on a rectangle with its length not larger than twice its width (non elongated geometry), the binary scheme is implemented by subdividing the two variable ranges of every subdomain of level $L-1$ by a factor two, thus generating four "children" subdomains of level $L$, for $L=0$ to $M$.

After a pre-processing phase devoted to this hierarchical subdivision, the multilevel algorithm starts with the PO surface integration over each of the elemental subdomains (or "patches") of level $M$. Due to the size of these patches, their radiated patterns in self-centered coordinate systems (i.e., with the origin at the center of the smallest sphere circumscribing the considered patch) are fully described by sampling the directions of observation very coarsely, according to Property 1. In the MLPO algorithm, we use the same grid of directions $\left\{\theta_{i}^{L}, \phi_{j}^{L}\right\}$ with $i=1, \ldots, N_{\theta}^{L}$ and $j=1, \ldots, N_{\phi}^{L}$, to sample the patterns of all patches at a given level. $N_{\beta}^{L}$ denotes the number of samples along the angular variable $\beta$ at level $L$. At level $M$, we take $N_{\theta}^{M}=N_{\theta}(N)$ and $N_{\phi}^{M}=N_{\phi}(N)$, using $N=k R^{M}$ in formula (3). For successively decreasing levels, we take $N_{\beta}^{L}=N_{\beta}^{M} 2^{M-L}$. It was shown in [10] that starting with a sufficiently sampled coarse grid at level $M$ and doubling the number of points in the grid for each of the variables $\theta$ and $\phi$ 
leads to sufficient sampling at all levels since $R^{L-1} \leq 2 R^{L}$.

The gist of the MLPO algorithm is the multilevel aggregation of partial patterns, which successively computes fields radiated by level $L-1$ subdomains from fields radiated by level $L$ subdomains, for $L=M$ to 1 . Let us consider the level $L$ subdomains $S_{n}^{L}$ (with $n=1$ to 4 in the simple conventional algorithm), which are "children" of the same level $L-1$ subdomain $S_{m}^{L-1}$. The "children" patterns will be aggregated to obtain the pattern radiated by their "parent" patch $S_{m}^{L-1}$, but this "aggregation" is not a simple addition:

- The sampling density of the "parent" pattern must be increased with respect to that of the "children" patterns, due to their respective sizes (Property 1). In a binary scheme, the sampling density along each angular variable is doubled. A low order interpolation algorithm can be used to that end, if the "children" subdomains patterns are computed in their respective self-centered coordinate systems, ensuring that they vary slowly with respect to observation directions (Property 1).

- To allow for "children" pattern summations, Property 2 is used to express the patterns of "sibling" subdomains (i.e. subdomains with the same "parent") in their common "parent" coordinate system.

The aggregation of the patterns of "sibling" subdomains is thus performed in three steps: (a) interpolation of the "children" patterns, (b) expression of the "children" patterns in the "parent" coordinate systems, (c) summation of the resulting "children" patterns.

The asymptotic complexity of the whole algorithm was demonstrated to be of $O\left(N^{2} \log N\right)$ with $N$ the linear dimension of the radiating surface with respect to the wavelength $[6,10]$.

\section{MLPO algorithm generalization}

A multireflector system such as the RATAN-600 S+F antenna system cannot be analyzed by simply using the conventional algorithm described above. Firstly, the fields radiated by the whole antenna system can be considered, in a first approximation, as being radiated by the primary feed to a first reflector, then successively radiated by a reflector to the "following" one, until the final reflexion towards the sky. Physical Optics integrals can be used to compute fields radiated by a reflector onto another one, if the non far field expression of these integrals is used. MLPO however relies on the validity of the "sampling theorem", and on the use of successive interpolations to retrieve large surface radiated fields from those radiated by its elemental subdomains. The validity of this approach in the non far field case will be examined. Secondly, oblong reflector surfaces impose constraints both on radiating surface multilevel subdivision and on observation domain sampling (reflector surfaces are both radiating and "observation" surfaces in multi-reflector systems). We present in the following subsections generalizations of the MLPO algorithm which address these constraints.

\subsection{Non far field formulation}

Let us consider a surface $S^{\prime}$ radiating fields on the surface $S$ which is not situated in its far field, and which will be subsequently considered as a radiating surface. In such a context, the PO or MLPO algorithm must compute the magnetic fields radiated by $S^{\prime}$ on a sufficiently sampled grid of points on the surface $S$.

It must be noted that in all cases, $S$ is in the far field of the level $M$ subdomains of the surface $S^{\prime}$, due to their small size (linear size of the order of a wavelength). The PO integrals on these subdomains of the surface $S^{\prime}$ can thus be performed using the far field expressions given in (1) and (2), the magnetic field being easily deduced from the electric field. 
Below a certain level of subdivision of the surface $S^{\prime}$, however, subdomains are too large for the surface $S$ to be in their far field. The following approximation will be used to express the magnetic fields on $S$ :

$$
\begin{gathered}
\boldsymbol{H}(\boldsymbol{r})=\int_{S^{\prime}} \boldsymbol{h}\left(\boldsymbol{r}, \boldsymbol{r}^{s}\right) \frac{e^{-j k\left|\boldsymbol{r}-\boldsymbol{r}^{s}\right|}}{\left|\boldsymbol{r}-\boldsymbol{r}^{s}\right|} d s \\
\text { with } \boldsymbol{h}\left(\boldsymbol{r}, \boldsymbol{r}^{s}\right)=-\frac{j k}{2 \pi} \widehat{\boldsymbol{r}} \times\left[\widehat{\boldsymbol{n}}\left(\boldsymbol{r}^{s}\right) \times \boldsymbol{H}^{s}\left(\boldsymbol{r}^{s}\right)\right]\left(1+\frac{1}{j k\left|\boldsymbol{r}-\boldsymbol{r}^{s}\right|}\right)
\end{gathered}
$$

Notations are similar to the ones previously introduced, with $\boldsymbol{r}$ the vector position of a point on $S$ and $\widehat{\boldsymbol{r}}$ the unit vector directed from a source point on $S^{\prime}$ with position vector $\boldsymbol{r}^{s}$ to an observation point on $S$ : $\widehat{\boldsymbol{r}}=\frac{\boldsymbol{r}-\boldsymbol{r}^{s}}{\left|\boldsymbol{r}-\boldsymbol{r}^{s}\right|}$.

The "sampling theorem" which is at the root of Property 1 is also valid for sampling fields on surfaces which are not located in the far field of sources [8,9]. The basic principle of the MLPO algorithm, consisting of coarsely sampling the fields radiated by the elemental subdomains, then hierarchically aggregating the subdomain fields at all levels, thus remains pertinent.

However, the magnetic field given by (4) exhibits not only phase variations with respect to the observation point position $\boldsymbol{r}$, as is the case for far field patterns, but also amplitude variations, which cannot be compensated by expressing the subdomain radiated fields in their self-centered coordinate systems. A new "compensated field", denoted by $\widetilde{\boldsymbol{H}}(\boldsymbol{r})$, is thus introduced as an intermediate set of vector values which can be easily interpolated. Considering the magnetic field radiated by the level $L$ subdomain $S_{m}$, with center $\boldsymbol{r}_{m}$ and "radius" $R_{m}$, this new "field" is given by:

$$
\widetilde{\boldsymbol{H}}\left(\boldsymbol{r}, \boldsymbol{r}_{m}\right)=\tilde{r}\left(\boldsymbol{r}, \boldsymbol{r}_{m}\right) e^{j k \tilde{r}\left(\boldsymbol{r}, \boldsymbol{r}_{m}\right)} \boldsymbol{H}(\boldsymbol{r}) \quad \text { with } \tilde{r}\left(\boldsymbol{r}, \boldsymbol{r}_{m}\right)=\sqrt{\left|\boldsymbol{r}-\boldsymbol{r}_{m}\right|^{2}+R_{m}^{2} / 2}
$$

This "compensated field" is defined at all points of the observation grid of level $L$, represented by the position vector $\boldsymbol{r}$ in the formula, and it is easily interpolated to the points of the grid of level $L-1$. Magnetic field values on the latter grid are then obtained by restoring the phase and amplitude variations as follows:

$$
\boldsymbol{H}(\boldsymbol{r})=\frac{e^{-j k \tilde{r}\left(\boldsymbol{r}, \boldsymbol{r}_{m}\right)}}{\tilde{r}\left(\boldsymbol{r}, \boldsymbol{r}_{m}\right)} \widetilde{\boldsymbol{H}}\left(\boldsymbol{r}, \boldsymbol{r}_{m}\right)
$$

where the position vector $\boldsymbol{r}$ now represents the points of the observation grid of level $L-1$. The validity of this "non far field" MLPO approach has already been checked in the case of a dual reflector antenna [11].

\subsection{Oblong reflector surface}

In the following, we consider as a special case of oblong geometry, a reflector surface which projects on an oblong rectangle, i.e. with its length larger than twice its width. In such a case, the radius of the smallest sphere circumscribing the surface is of the order of the rectangle half-length $L_{0} / 2$, independent of the rectangle width $l_{0}$. Application of the simple binary scheme used in the conventional MLPO algorithm leads to a number of subdivision levels $M$ with $L_{0} / 2^{M}$ of the order of $\lambda$, to a number of subdivisions of the order of $L_{0}^{2}$ at level $M$, and to an asymptotic complexity of $O\left(N_{0}^{2} \log N_{0}\right)$ with $N_{0}$ the electrical size of the reflector surface.

An alternative subdivision scheme consists in subdividing only along the rectangle length until the subdomains are no longer elongated, proceeding further with the conventional scheme. In this way, the number of subdomains at the highest level $M$ is equal to $2^{M+M^{\prime}}$ where $L_{0} / 2^{M}$ and $l_{0} / 2^{M^{\prime}}$ of the order of $\lambda$. The number of subdomains of level $M$ is thus reduced by a factor roughly equal to $L_{0} / l_{0}$, by comparison with the conventional scheme, and so are the number of operations in the integration and 
aggregation phases of the MLPO algorithm. The asymptotic complexity of the MLPO algorithm in that case is $O\left(\alpha N_{0}^{2} \log N_{0}\right)$ with $\alpha=l_{0} / L_{0}$ the inverse of the rectangle elongation factor.

Also, when the MLPO algorithm is used to compute fields radiated on a reflector surface of oblong shape, the oversampling along the variable describing the "short" dimension is large with respect to the sufficient one, for the highest levels. Interpolating along the other variable only, for these levels, until obtaining oversampling values of the same order for both variables, leads to reduced algorithm complexity and memory savings.

\section{Numerical results obtained for the RATAN-600 $\mathrm{S}+\mathrm{F}$ system}

This section presents results illustrating the use of the MLPO algorithm for a multireflector system involving extremely large reflectors with elongated geometry. The presented results have been obtained for the sub-system composed of the feed, the secondary reflector, and the main reflector of the RATAN-600 South sector antenna system (cf. description in the Introduction, and Figs. 1 to 3).

The computations are performed for an operating wavelength of $\lambda=8 \mathrm{~mm}$, corresponding to $37.5 \mathrm{GHz}$, in the $33-38 \mathrm{GHz}$ band of interest. A complex source point with collimation distance $b=2.44 \mathrm{~mm}$ and horizontal polarization is used to represent the feed, its Gaussian radiation pattern being very similar to the exact feed pattern. The elevation angle of the source beam axis is $65^{\circ}$, yielding an approximate $-10 \mathrm{~dB}$ taper along the top and bottom edges of the secondary reflector. Fig. 4 presents a map of the magnitude of the tangent magnetic field on the surface of the secondary reflector, as a function of the $x$ horizontal and $y$ vertical coordinates in a projected vertical plane.

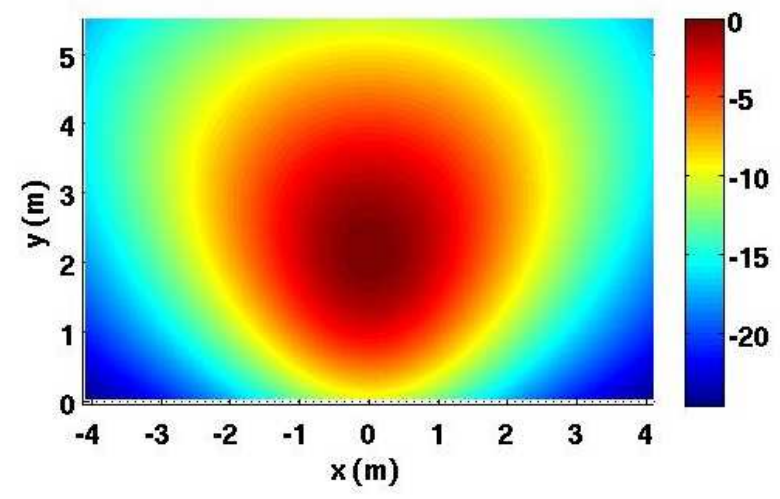

Figure 4. Tangent magnetic field magnitude on the surface of the secondary reflector (represented in a projected vertical plane).

The radius of the smallest sphere circumscribing the secondary reflector is equal to $655 \lambda$ for $\lambda=8 \mathrm{~mm}$. As can be seen on Figs. 2 and 3, the reflector surface projects in a vertical plane on a rectangle of horizontal and vertical dimensions $8.2 \mathrm{~m}$ and $5.5 \mathrm{~m}$, respectively. The number of decomposition levels is $M=10$ along both dimensions, yielding subdomains of radii ranging between $0.6 \lambda$ and $0.75 \lambda$ at level 10. The PO integrals on these subdomains use a 6 -point Gauss quadrature rule along each variable. The "non far field" formulations are used for these PO integrals, as well as for the multilevel aggregation in the MLPO algorithm.

The main reflector surface, which projects on a rectangle of horizontal and vertical dimensions $330 \mathrm{~m}$ and $7.4 \mathrm{~m}$, respectively, is clearly of oblong geometry. From the focus of the secondary reflector, this 
surface is seen under angles of $120^{\circ}$ in azimuth and $3^{\circ}$ in elevation. The coarse observation grid on the main reflector (used to represent the magnetic fields radiated by the secondary reflector subdomains of level 10) comprises 16 (resp. 1) point(s) along the "long" horizontal axis $x$ (resp. "short" vertical axis $y$ ). The final grid of points on the main reflector surface comprises 16384 points along $x$ and 256 points along $y$. The interval between two neighboring point coordinates in the coarse grid is $20.625 \mathrm{~m}$ along $x$ and $7.4 \mathrm{~m}$ along $y$; in the final fine grid, these intervals are $2.01 \mathrm{~cm}$ and $2.89 \mathrm{~cm}$ along $x$ and $y$, respectively. These grids correspond to oversampling values of 9.8 and 24.5 along $x$ and $y$ respectively, at level 10 . The multilevel algorithm is optimized (cf. last paragraph of subsection 3.2) to reduce the oversampling along the short dimension ( $y$ variable): interpolations at levels 10 and 9 are performed only along $x$, yielding oversampling values of 11.3 along $x$ and 7.1 along $y$ in the final grid (level 0 ).

Fig. 5 shows the magnitude of the magnetic field computed with the MLPO algorithm on the main reflector surface, represented in a projected vertical plane. The measured computation time on a single processor Intel X5472 @ 3.0GHz is of about one hour. Diffraction effects are visible on this map, contrary to the results previously obtained via Geometrical Optics. In Fig. 6 are presented the variations of the magnetic field components along the wide horizontal dimension on the main reflector, as a function of the variable $x$ in the projected plane, at mid-height of the reflector $(y=2.75 \mathrm{~m})$. These curves clearly exhibit the influence of the finite size of the secondary reflector, yielding diffraction of the feed radiation. Accurate information on field polarization can be especially useful to optimize complex focal systems, as will be the case for the millimetric range extension of the RATAN-600 S+F antenna system (cf. Section 1).

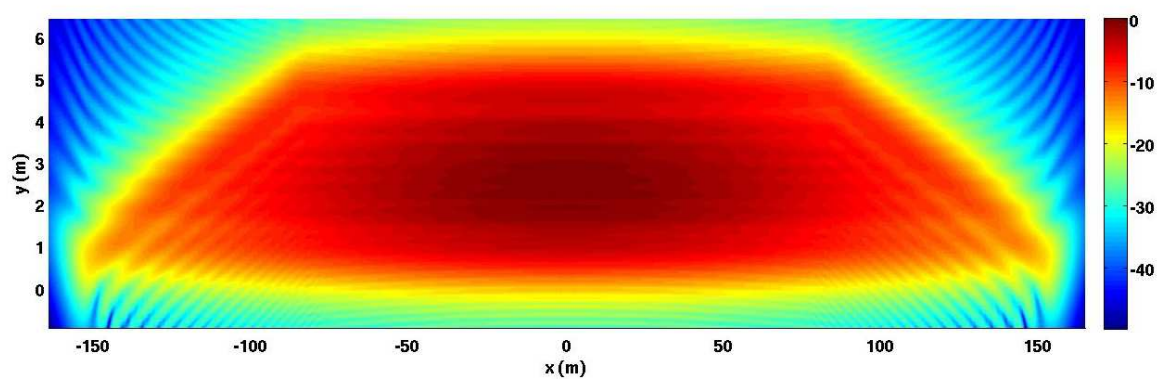

Figure 5. Magnetic field magnitude on the surface of the main reflector (represented in a projected vertical plane).

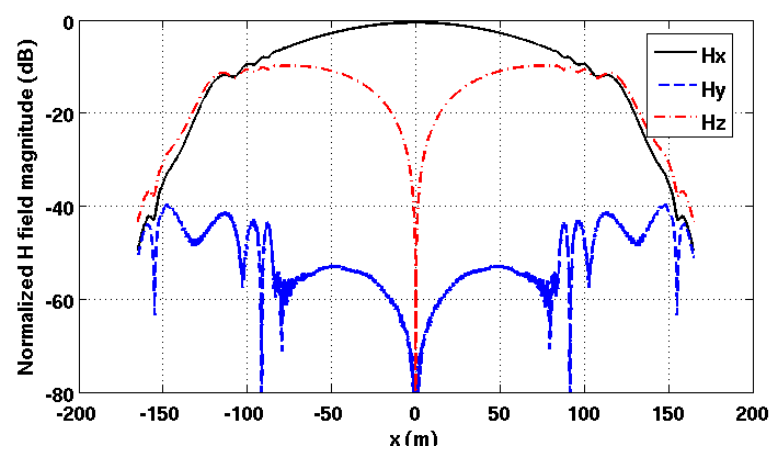

Figure 6. Magnitude of the magnetic field cartesian components on the surface of the main reflector, along the mid-height horizontal line $(y=2.75 \mathrm{~m})$ in a projected vertical plane. 


\section{Conclusion}

The MLPO algorithm is a fast Physical Optics algorithm allowing to evaluate PO integrals over large arbitrary shaped surfaces with an asymptotic complexity comparable to that of the FFT-based techniques. The algorithm has been adapted to the case of complex antenna systems comprising very large surfaces with oblong shape. The non far field version of the algorithm, which had already been validated for moderate size dual reflector antennas, has been used to analyze a sub-system of the extremely large RATAN-600 $\mathrm{S}+\mathrm{F}$ antenna system in the millemetric range, providing accurate information about depolarization as well as diffraction effects.

The next steps in this work will aim at simulating the far field pattern of the antenna system without and with the periscope reflector, and finally of the extended system with tertiary reflector and focal receiver array. The MLPO algorithm was shown to be well suited for parallelization, which is an elegant way of addressing the problem of memory requirements in problems involving very large radiating surfaces, while accelerating further the execution [12]. A parallelized implementation of the algorithm used to analyze the RATAN-600 antenna system would then allow for better optimization of the long focal multireceiver focal system, as well as for full 3D pattern simulations, including spillover and PTD contributions.

\section{References}

[1] Y. Pariiskii, D. Korol'kov, Experiment COLD: the first deep sky survey with the RATAN-600 radio telescope, Sov. Sci. Rev. E Astrophys. Space Phys. 5 (1986) 39-179.

[2] Y. Pariiskii, RATAN-600: The world's biggest reflector at the "cross roads", IEEE Antennas Propagat. Mag. 35 (1993) $7-12$.

[3] M. Lebedev, V. Khaikin, A. Boag, C. Letrou, Optical and diffraction simulation techniques for large multibeam reflector, in: MSMW10: Seventh IEEE International Kharkov Symposium on Physics and Engineering of Microwaves, Millimeter and Submillimeter Waves, Kharkov, Ukraine, 2010, pp. 1-3.

[4] E. Majorova, Computation of the RATAN-600 radio telescope power beam pattern with allowance for diffraction effects in the southern-sector-with-flat-reflector mode, Astrophysical Bulletin 66 (2011) 215-232.

[5] K. Pontoppidan, ed., TICRA, Technical Description of GRASP9, www.ticra.com (2005).

[6] A. Boag, C. Letrou, Fast Physical Optics algorithm for lens and reflector antennas, IEEE Trans. Antennas Propagat. 51 (2003) 1063-1068.

[7] C. Letrou, A. Boag, Generalized multilevel Physical Optics (MLPO) for comprehensive analysis of reflector antennas, IEEE Trans. Antennas Propagat. (accepted for publication).

[8] O. Bucci, G. Franceschetti, On the spatial bandwidth of scattered fields, IEEE Trans. Antennas Propagat. 35 (1987) $1445-1455$.

[9] O. Bucci, C. Gennarelli, C. Savarese, Representation of electromagnetic fields over arbitrary surfaces by a finite and nonredundant number of samples, IEEE Trans. Antennas Propagat. 46 (3) (1998) 351-359.

[10] A. Boag, C. Letrou, Multilevel Fast Physical Optics algorithm for radiation from non-planar apertures, IEEE Trans. Antennas Propagat. 53 (2005) 2064-2072.

[11] C. Letrou, A. Boag, Analysis of very large dual-reflector antennas using multilevel Physical Optics algorithm, in: Proc. ICEAA'07: International Conference on Electromagnetics in Advanced Applications, Torino, Italy, 2007.

[12] C. Parrot, D. Millot, C. Letrou, A. Boag, Scaling of the multilevel Physical Optics parallel algorithm on a large grid, in: EuCAP 2010 : Proc. 4th European Conference on Antennas and Propagation, Barcelona, Spain, 2010, pp. 1-4. 